\title{
Algebraic cone metric spaces and fixed point theorems of contractive mappings
}

\author{
Mohammad Akbari Tootkaboni ${ }^{1}$ and Alireza Bagheri Salec ${ }^{2 *}$
}

*Correspondence:

alireza_bagheri_salec@yahoo.com

${ }^{2}$ Department of Mathematics, Qom University, Qom, Iran

Full list of author information is

available at the end of the article

\begin{abstract}
In this paper we introduce algebraic cone metric spaces, prove some fixed point theorems of contractive mappings on algebraic cone metric spaces, and improve some well-known results in the non-normal case.
\end{abstract}

Keywords: cone metric space; Banach principle contraction; fixed point

\section{Introduction and preliminary}

There exist a number of generalizations of metric spaces. One of them is the cone metric space initiated by Huang and Zhang [1]. They described the convergence in cone metric spaces, introduced the notion of completeness and proved some fixed point theorems of contractive mappings on these spaces. Let us recall some notions and definitions.

Let $\mathcal{A}$ be a real Banach space and $\mathcal{P}$ be a subset of $\mathcal{A}$. $\mathcal{P}$ is called a cone if and only if

(i) $\mathcal{P}$ is closed and nonempty and $\mathcal{P} \neq\{\theta\}$,

(ii) if $a, b \in \mathbb{R} ; a, b \geq 0 ; x, y \in \mathcal{P}$, then $a x+b y \in \mathcal{P}$,

(iii) if $x \in \mathcal{P}$ and $-x \in \mathcal{P}$, then $x=\theta$.

Given a cone $\mathcal{P} \subseteq \mathcal{A}$, a partial ordering $\preceq$ with respect to $\mathcal{P}$ is defined by $x \preceq y$ if $y-x \in \mathcal{P}$. Furthermore, we write $x \prec y$ if $x \preceq y$ and $x \neq y$, while $x \ll y$ will stands for $y-x \in \operatorname{int} \mathcal{P}$, where int $\mathcal{P}$ is the interior of $\mathcal{P}$.

The cone $\mathcal{P}$ is called normal if there is a number $M>0$, such that for every $x, y \in \mathcal{A}$, $\theta \preceq x \preceq y$ implies $\|x\| \leq M\|y\|$. The least positive number satisfying the above condition is called the normal constant of $\mathcal{P}$.

Lemma 1.1 The following conditions are equivalent for a cone $\mathcal{P}$ in the Banach space $(\mathcal{A},\|\cdot\|)$ :

(a) $\inf \{\|x+y\|: x, y \in \mathcal{P},\|x\|=\|y\|=1\}>0$.

(b) $\mathcal{P}$ is a normal cone.

(c) For arbitrary sequences $\left\{x_{n}\right\},\left\{y_{n}\right\}$ and $\left\{z_{n}\right\}$ in $E$, if $x_{n} \preceq y_{n} \preceq z_{n}$ for each $n$ and $\lim _{n \rightarrow \infty} x_{n}=\lim _{n \rightarrow \infty} z_{n}=x$, then $\lim _{n \rightarrow \infty} y_{n}=x$.

(d) There exists a norm $\|\cdot\|_{1}$ on $\mathcal{A}$, equivalent to the given norm $\|\cdot\|$, such that the cone $\mathcal{P}$ is monotone with respect to $\|\cdot\|_{1}$, i.e., if $\theta \preceq x \preceq y$, then $\|x\|_{1} \leq\|y\|_{1}$.

Proof See [2-4], and [5].

Definition 1.2 Let $X$ be a nonempty set, and let $\mathcal{A}$ be a real Banach space and $\mathcal{P} \subseteq \mathcal{A}$ be a cone. Suppose the mapping $d: X \times X \rightarrow \mathcal{A}$ satisfies:

(2014 Tootkaboni and Bagheri Salec; licensee Springer. This is an Open Access article distributed under the terms of the Creative Commons Attribution License (http://creativecommons.org/licenses/by/2.0), which permits unrestricted use, distribution, and reproduction in any medium, provided the original work is properly cited. 
(i) $\theta \preceq d(x, y)$ for every $x, y \in X$ and $d(x, y)=\theta$ if and only if $x=y$;

(ii) $d(x, y)=d(y, x)$ for every $x, y \in X$;

(iii) $d(x, y) \preceq d(x, z)+d(z, y)$ for every $x, y, z \in X$.

Then $d$ is called a cone metric on $X$ and $(X, d)$ is called a cone metric space.

Let $(X, d)$ be a cone metric space, $\left\{x_{n}\right\}$ be a sequence in $X$, and $x \in X .\left\{x_{n}\right\}$ is said to be convergent to $x$, if for every $\theta \ll c$ there is $N \in \mathbb{N}$, such that for all $n>N, d\left(x_{n}, x\right) \ll c$. Likewise, $\left\{x_{n}\right\}$ is called a Cauchy sequence in $X$ if for every $\theta \ll c$ there is $N \in \mathbb{N}$, such that for each $n, m>N, d\left(x_{n}, x_{m}\right) \ll c$. A cone metric space $X$ is said to be complete if every Cauchy sequence in $X$ is convergent in $X$.

To replace the standard properties of a metric, the properties in the following lemma of cone metrics are often useful while dealing with cone metrics when the cone is not normal.

Lemma 1.3 Let $(X, d)$ be a cone metric space with corresponding cone $\mathcal{P}$. If $x \in \mathcal{P}$ and $\left\{x_{n}\right\}$ and $\left\{a_{n}\right\}$ are sequences in $X$ and $\mathcal{A}$, respectively, then:

(a) If $\theta \preceq x \ll c$ for all $c \in$ int $\mathcal{P}$, then $x=\theta$.

(b) If $\theta \preceq d\left(x_{n}, x\right) \preceq a_{n}$ and $a_{n} \rightarrow \theta$, then for each $c \in \operatorname{int} \mathcal{P}$ there exists a natural number $n_{0}$, such that $d\left(x_{n}, x\right) \ll c$ for each $n>n_{0}$.

(c) If $c \in \operatorname{int} \mathcal{P}$ and $a_{n} \rightarrow \theta$, then there exists a natural number $n_{0}$, such that for every $n>n_{0}$, we have $a_{n} \ll c$.

Proof See [3], p.2598.

It follows from Lemma 1.3(c) that the sequence $\left\{x_{n}\right\}$ converges to $x \in X$ if $d\left(x_{n}, x\right) \rightarrow \theta$, and $\left\{x_{n}\right\}$ is a Cauchy sequence if $d\left(x_{n}, x_{m}\right) \rightarrow \theta$ as $n, m \rightarrow \infty$. The converse is true if $\mathcal{P}$ is a normal cone. To see that $x_{n} \rightarrow x$ does not necessarily imply $d\left(x_{n}, x\right) \rightarrow \theta$ at non-normal cones, see example (e) in Section 4.

There are several states of cone metric spaces. For example, after cone metric spaces over topological vector spaces, in [6] the authors introduce the concept of tvs-cone $b$-metric spaces over a solid cone. In the following section another state of metric spaces is presented: an algebraic cone metric space.

\section{Algebraic cones and Banach cone algebras}

The concept of cone metric spaces over Banach algebras was previously introduced by Liu and $\mathrm{Xu}$ in [7]. They proved some fixed point theorems of generalized Lipschitz mappings with weaker conditions on the generalized Lipschitz constant $k$ by means of the spectral radius (see Theorems 2.1, 2.2, and 2.3 in [7]). Indeed for the mapping $T: X \rightarrow X$ the generalized Lipschitz condition is

$$
d(T x, T y) \leq k d(x, y)
$$

for all $x, y \in X$, where $k \in P$ with $\rho(k)<1$.

The same authors in [8] introduced the concept of quasicontractions on cone metric spaces over Banach algebras and proved the existence and uniqueness of fixed points of such mapping. Afterwards Xu and Radenović deleted the superfluous assumption of normality in [7] (see Theorems 3.1, 3.2, and 3.3 in [9]). 
In this section, we will present the definition of the algebraic cones, Banach cone algebras, and some properties related to this concept.

Definition 2.1 ( $c f .[10,11]$ and $[12])$ Let $\mathcal{P} \subseteq \mathcal{A}$ be a cone, and $X$ be a vector space over $\mathbb{C}$. Suppose the mapping $\|\cdot\|: X \rightarrow \mathcal{P}$ satisfies:

(N1) $\|x\|=\theta_{\mathcal{A}}$ if and only if $x=\theta_{X}$,

(N2) $\|x+y\| \preceq\|x\|+\|y\|$, for every $x, y \in X$,

(N3) $\|k x\|=|k|\|x\|$ for every $k \in \mathbb{C}$ and $x \in X$.

Then $\|\cdot\|$ is called a cone norm on $X$, and the pair $(X,\|\cdot\|)$ is called a cone normed space (CNS).

Sometimes, to emphasize the cone $\mathcal{P}$, we use $\|x\|_{\mathcal{P}}$ instead of $\|x\|$.

A real topological algebra is an algebra $\mathcal{A}$ over $\mathbb{F}$ furnished with a topology $\tau$ such that

(i) the map $(x, y) \mapsto x+y: \mathcal{A} \times \mathcal{A} \rightarrow \mathcal{A}$ is continuous,

(ii) the map $(t, x) \mapsto t x: \mathbb{R} \times \mathcal{A} \rightarrow \mathcal{A}$ is continuous,

(iii) the map $(x, y) \mapsto x y: \mathcal{A} \times \mathcal{A} \rightarrow \mathcal{A}$ is continuous.

When $\mathbb{F}=\mathbb{C}, \mathcal{A}$ is called a complex topological algebra and if $\mathbb{F}=\mathbb{R}, \mathcal{A}$ is called a real topological algebra.

It is obvious that every Banach algebra is a topological algebra.

Definition 2.2 Let $\mathcal{A}$ be a Banach algebra with identity element $e_{\mathcal{A}}$ and $\mathcal{P} \subseteq \mathcal{A}$ be a cone. $\mathcal{P}$ is an algebraic cone if $e_{\mathcal{A}} \in \mathcal{P}$, and for each $a, b \in \mathcal{P}, a b \in \mathcal{P}$.

Therefore, for each $x, y \in \mathcal{A}$ and $a \in \mathcal{P}$ if $x \preceq y$ and $a \in \mathcal{P}$, then $a x \preceq a y$.

For example if $\mathcal{A}$ is a $C^{*}$-algebra and $\mathcal{A}_{+}$is its positive elements, i.e. the set of all Hermitian element $a \in \mathcal{A}$ with $\sigma(a) \subseteq \mathbb{R}_{+}$, then $\mathcal{A}_{+}$is a cone in $\mathcal{A}$, and whenever $\mathcal{A}$ is commutative, $\mathcal{A}_{+}$is an algebraic cone.

Definition 2.3 Let $X$ be an algebra, $\mathcal{A}$ be a real topological algebra (or as a special case be a Banach algebra), and $\mathcal{P}$ be an algebraic cone in $\mathcal{A}$. Furthermore, let $\|\cdot\|: X \rightarrow \mathcal{A}$ be a cone norm, such that $(X,\|\cdot\|)$ is a Banach cone metric space, and $\|x y\| \preceq\|x\|\|y\|$ for each $x, y \in X$. Then we say that $(X,\|\cdot\|)$ is a cone Banach cone algebra.

In the following, $X$ is an algebra with identity $e_{X}$ and $\left(\mathcal{A},\|\cdot\|_{\mathcal{A}}\right)$ is a Banach algebra with identity $e_{\mathcal{A}}$. Moreover, in Section 4 , some examples are presented in the topological algebra case.

Theorem 2.4 Suppose $(X,\|\cdot\|)$ is a normal Banach cone algebra, $x \in X$ and \|\|$x\|\|_{\mathcal{A}}<1$, then

(a) $e_{X}-x$ is invertible,

(b) \|\|$\left(e_{X}-x\right)^{-1}-e-x\|\|_{\mathcal{A}} \leq \frac{\|\| x\|\|_{\mathcal{A}}^{2}}{1-\|\| x\|\| \|_{\mathcal{A}}}$

(c) \|\|$\phi(x)\|\|_{\mathcal{A}}<1$ for every homomorphism $\phi: X \rightarrow \mathcal{A}$, such that \|\|$\phi\left(e_{X}\right)\|\|_{\mathcal{A}}=1$.

Proof (a) Since $\left\|x^{n}\right\| \leq\|x\|^{n}$ and \|\|$x\|\|_{\mathcal{A}}<1$, the elements

$$
s_{n}=e_{X}+x+x^{2}+\cdots+x^{n}
$$


form a Cauchy sequence $X$ by the normality of $\mathcal{P}$. Since $X$ is complete, there exists $s \in X$, such that $s_{n} \rightarrow s$. As $x^{n} \rightarrow \theta$ and $s_{n} \cdot\left(e_{X}-x\right)=e_{X}-x^{n+1}=\left(e_{X}-x\right) \cdot s_{n}$, the continuity of multiplication implies that $s$ is the inverse of $e_{X}-x$.

(b) \|\|$s-e_{X}-x\|\|_{\mathcal{A}}=\|\| x^{2}+x^{3}+\cdots\|\|_{\mathcal{A}} \leq \sum_{n=2}^{\infty}\|\| x\left\|^{n}\right\|_{\mathcal{A}}=\frac{\|\| x\|\|_{\mathcal{A}}^{2}}{1-\|\| x\|\|_{\mathcal{A}}}$.

(c) Suppose $\lambda \in \mathbb{C},|\lambda| \geq 1$. By (a) $e_{X}-\lambda^{-1} x$ is invertible. So $\phi\left(e_{X}\right)-\lambda^{-1} \phi(x)=\phi\left(e_{X}-\right.$ $\left.\lambda^{-1} x\right) \neq \theta$. Hence

$$
\|\| \phi(x)\|\|_{\mathcal{A}} \neq \lambda\|\| \phi\left(e_{X}\right)\|\|_{\mathcal{A}}=\lambda
$$

This completes the proof.

Note that if the condition \|\|$x\|\|_{\mathcal{A}}<1$ in Theorem 2.5 is replaced by $\sum_{n=1}^{\infty}\|x\|^{n} \leq 1$, then (a) is still true. In the next theorem, let $G(X)$ be the group of invertible elements of $X$.

Theorem 2.5 If $X$ is a Banach cone algebra on $\mathcal{P}$, then $G(X)$ is open in $X$ and the mapping $x \mapsto x^{-1}: G(X) \rightarrow G(X)$ is a homeomorphism.

Proof Let $x \in G(X)$ and $h \in X$. If $\left\|x^{-1} h\right\| \leq \frac{1}{2}$, then by Theorem 2.4, $e+x^{-1} h$ and $x+h$ belong to $G(X)$ (notice that $x+h=x\left(e_{X}+x^{-1} h\right)$ ) and $\left\|x^{-1} h\right\|^{-1}$ is contained in $G(X)$. Also by Theorem 2.4,

$$
\begin{aligned}
\|\|(x+h)^{-1}-x^{-1}+x^{-1} h x^{-1}\|\|_{\mathcal{A}} & =\|\|\left(\left(e_{X}+x^{-1} h\right)^{-1}-e_{X}+x^{-1} h\right) x^{-1}\|\|_{\mathcal{A}} \\
& \leq 2\|\| x^{-1}\|\|_{\mathcal{A}}^{3}\|\| h\|\|_{\mathcal{A}}^{2} .
\end{aligned}
$$

Therefore, \|\|$(x+h)^{-1}-x^{-1}\|\|_{\mathcal{A}} \leq\|\| h\|\| \|_{\mathcal{A}}\left(\|\| x^{-1}\|\|_{\mathcal{A}}^{2}+2\|\| x^{-1}\|\|_{\mathcal{A}}^{3}\|\| h\|\|_{\mathcal{A}}\right)$. Hence the mapping $x \mapsto x^{-1}: G(X) \rightarrow G(X)$ is continuous and homeomorphism.

Note that for each $x \in X$, the spectrum $\sigma(x)$ of $x$ is the set of all complex numbers $\lambda$, such that $\lambda e-x$ is not invertible. The complement of $\sigma(x)$ is the resolvent set of $x$. It consists of all $\lambda \in G$ for which there exists $\left(\lambda e_{X}-x\right)^{-1}$.

The spectral radius of $x$ is the number $\rho(x)=\sup \{|\lambda|: \lambda \in \sigma(x)\}$. For every $x \in X$, and $\lambda \in \mathbb{C}$ if $|\lambda| \geq\|\| x\|\|_{\mathcal{A}}$ then $e_{X}-\lambda^{-1} x$ lies in $G(X)$, by Theorem 2.4, and so does $\lambda e-x$. Thus, $\lambda \notin \sigma(x)$. This proves that \|\|$x\|\|_{\mathcal{A}}$ is an upper bound for $\sigma(x)$, and $\rho(x) \leq\|\| x\|\|_{\mathcal{A}}$. In addition by [13], there exists a norm $\|\cdot\|_{\mathcal{A}}^{\prime}$ on $\mathcal{A}$, such that $\mathcal{P}$ in $\left(\mathcal{A},\|\cdot\|_{A}^{\prime}\right)$ is a normal cone with constant normal 1 . Hence we can assume that $K=1$. So $\sigma(x)$ is compact and

$$
\rho(x)=\lim _{n \rightarrow \infty}\|\| x^{n}\|\|_{\mathcal{A}}^{\frac{1}{n}}=\inf _{n \geq 1}\|\| x^{n}\|\|_{\mathcal{A}}^{\frac{1}{n}}
$$

(see Theorem 10.13 in [14]). Since the combination $\|\cdot\|_{\mathcal{A}}$ with $\|\cdot\|$ is a real-valued norm, we can present an extension of the Gelfand-Mazur theorem.

Theorem 2.6 (Gelfand-Mazur) If $X$ is a normal Banach cone algebra in which every nonzero element is invertible, then $X$ is isometrically isomorphic to the complex field.

\section{Fixed point theorems in algebraic cone metric spaces}

In this section some fixed point theorems of generalized Lipschitz mappings with weaker conditions than the condition $\rho(k)<1$, are proved (see Definition 3.1 for the condition). 
Therefore some theorems in [7] and [9] are improved (see Theorems 3.3 and 3.9 for instance).

Let $(X, d)$ be an algebraic cone metric space and $F: X \rightarrow X$ be a self map. $F$ is called Lipschitzian if and only if there exists a constant $L \in \mathcal{P}$, called the Lipschitz coefficient for $F$, such that

$$
d(F(x), F(y)) \leq L d(x, y)
$$

for each $x, y \in X$. In a similar way, we can say that $F$ is a contraction if $\|L\|<1$. In the following, we will see that this definition of contraction maps is general.

When $(X, d)$ is a complete metric space, and $F$ is a contraction, we know that the statement ${ }^{\prime}|L|<1$ if and only if $\sum_{n=1}^{\infty}|L|^{n}<\infty$ ' has an important role in the proof of Banach fixed point theorem. But in a Banach algebra $\mathcal{A}$, the statement ${ }^{\prime}\|L\|<1$ if and only if $\sum_{n=1}^{\infty}\|L\|^{n}<$ $\infty^{\prime}$ is not true. In fact for $L \in \mathcal{A}, \sum_{n=1}^{\infty}\|L\|^{n}<\infty$ does not imply that $\|L\|<1$ (see example (f) in Section 4). For this reason, we have to state a scholastic definition of contraction.

Definition 3.1 Let $(X, d)$ be an algebraic cone metric space, and let $F: X \rightarrow X$ be a Lipschitzian map with Lipschitz coefficient $L$. $F$ is a contraction map if $\sum_{n=1}^{\infty}\|L\|^{n}<\infty$, i.e. the series $\sum_{n=1}^{\infty} L^{n}$ is absolutely convergent.

Let $L \in \mathcal{A}$ be a Lipschitz coefficient for a self-map $F$, then each of the properties $\rho(L)=$ $\lim _{n \rightarrow \infty}\left\|L^{n}\right\|^{\frac{1}{n}}<1$ and $\|L\|<1$ imply that $\sum_{n=1}^{\infty}\|L\|^{n}<\infty$, but example (f) of Section 4 shows that the converse cannot necessarily be established.

Lemma 3.2 Let $\mathcal{A}$ be a Banach algebra with a unit $e_{\mathcal{A}}$, and $L \in \mathcal{A}$. If $\sum_{n=1}^{\infty}\|L\|^{n}<\infty$, then $e_{\mathcal{A}}-L$ is invertible and $\left(e_{\mathcal{A}}-L\right)^{-1}=\sum_{n=0}^{\infty} L^{n}$.

Proof The proof is obvious.

Before presenting some fixed point theorems we remark that although each cone metric space over a solid cone is metrizable, as was shown by various methods, however, not all fixed point results can be reduced in this way to their standard metric counterparts. So this line of investigation is still open (see, e.g., [15]).

The next theorem is an extension of Theorem 2.1 in [7], Theorem 3.1 in [9] and Theorem 1.1 in [16].

Theorem 3.3 Let $(X, d)$ be a complete algebraic cone metric space and $F: X \rightarrow X$ be a contraction map with Lipschitz coefficient $L$. Then $F$ has a unique fixed point $u \in X$.

Furthermore, for each $x \in X$, we have $\lim _{n \rightarrow \infty} F^{n}(x)=u$ with

$$
d\left(F^{n}(x), u\right) \preceq L^{n}\left(e_{\mathcal{A}}-L\right)^{-1} d(x, F(x)) .
$$

Proof Since $F$ is a contraction with Lipschitz coefficient $L$, we have

$$
d(F(x), F(y)) \preceq L d(x, y)
$$

for each $x, y \in X$, and $\sum_{n=1}^{\infty} L^{n}$ is absolutely convergent. For existence, select $x \in X$. We first show that $\left\{F^{n}(x)\right\}$ is Cauchy sequence. Let $n \in \mathbb{N} \cup\{0\}$, then

$$
d\left(F^{n}(x), F^{n+1}(x)\right) \preceq L d\left(F^{n-1}(x), F^{n}(x)\right) \preceq \cdots \preceq L^{n} d(x, F(x)) .
$$


Thus for $m>n$,

$$
\begin{aligned}
d\left(F^{n}(x), F^{m}(x)\right) \preceq & d\left(F^{n}(x), F^{n+1}(x)\right)+d\left(F^{n+1}(x), F^{n+2}(x)\right) \\
& +\cdots+d\left(F^{m-1}(x), F^{m}(x)\right) \\
\preceq & L^{n} d(x, F(x))+\cdots+L^{m-1} d(x, F(x)) \\
\preceq & L^{n} d(x, F(x))\left(e_{\mathcal{A}}+L+\cdots+L^{k}+\cdots\right) \\
= & d(x, F(x)) L^{n}\left(e_{\mathcal{A}}-L\right)^{-1},
\end{aligned}
$$

that is, for $n \in \mathbb{N} \cup\{0\}, m \in \mathbb{N}$ and $m>n$,

$$
d\left(F^{n}(x), F^{m}(x)\right) \preceq L^{n}\left(e_{\mathcal{A}}-L\right)^{-1} d(x, F(x)) .
$$

By Lemma 1.3(b), this shows that $\left\{F^{n}(x)\right\}$ is a Cauchy sequence and, since $X$ is complete, there exists a $u \in X$ with $\lim _{n \rightarrow \infty} F^{n}(x)=u$. Hence by continuity $F(u)=u$. It is obvious that $u$ is unique.

For each $x \in X$ and $\theta \neq r \in \mathcal{P}$, let $B\left(x_{\circ}, r\right)=\left\{x \in X: d\left(x, x_{\circ}\right) \ll r\right\}$ and $B\left[x_{\circ}, r\right]=B\left(x_{\circ}, r\right) \cup$ $\left\{x \in X: d\left(x, x_{0}\right)=r\right\}$. Then the collection $\left\{B\left(x_{0}, r\right): x \in X, r \in\right.$ int $\left.\mathcal{P}\right\}$ forms a basis for the cone metric topology of $(X, d)$. Also it is easily seen that, for each $\theta \neq r \in \mathcal{P}, B\left(x_{0}, r\right)$ is an open set in cone metric space $(X, d)$. As in the real case, the equality $B\left[x_{\circ}, r\right]=\overline{B\left(x_{\circ}, r\right)}$ does not necessarily hold for all $x \in X$ and $\theta \neq r \in \mathcal{P}$. In the next lemma we express a necessary and sufficient condition for this equality.

Lemma 3.4 If $(X, d)$ is a cone metric space, then the following statements are equivalent.

(a) For each $x \in X$ and each $\theta \ll r, B[x, r]=\overline{B(x, r)}$.

(b) For each $x, y \in X$ and each $\theta \ll \epsilon$, there exists $z \in X$, such that $d(z, y) \ll \epsilon$ and $d(z, x) \ll d(x, y)$.

Proof (a) implies (b). If $x, y \in X$ and $x \neq y$, then $r=d(x, y) \in \mathcal{P}-\{\theta\}$. Now (a) implies that $y \in \overline{B(x, r)}$ and so for each $\theta \ll \epsilon$, there exists $z \in B(x, r)$ such that $d(y, z) \ll \epsilon$.

(b) implies (a). For each $y \in B[x, r]$, if $d(x, y) \ll r$ then $y \in B(x, r) \subseteq \overline{B(x, r)}$. If $d(x, y)=r$, then let $\epsilon_{n}=\frac{r}{n}$. So there exists $z_{n} \in X$ such that $d\left(z_{n}, y\right) \ll \epsilon_{n}$ and $d\left(x, z_{n}\right) \ll d(x, y)=r$. Hence there exists a sequence in $B(x, r)$ such that $\lim _{n \rightarrow \infty} z_{n}=y$. This implies $y \in \overline{B(x, r)}$.

Corollary 3.5 Let $(X,\|\cdot\|)$ be an algebraic cone metric space. Then for each $x \in X$ and each $r \succ \theta, B[x, r]=\overline{B(x, r)}$.

Theorem 3.6 Let $(X,\|\cdot\|)$ be a complete algebraic cone metric space, $r \in \operatorname{int} \mathcal{P}$ and $x_{\circ} \in X$. Suppose $F: B\left(x_{\circ}, r\right) \rightarrow X$ is a contraction with Lipschitz coefficient $L$, and

$$
d\left(F\left(x_{\circ}\right), x_{\circ}\right) \ll\left(e_{\mathcal{A}}-L\right) r \text { and }\|r\|<1 .
$$

Then $F$ has a unique fixed point in $B\left(x_{\circ}, r\right)$.

Proof There exists a $r_{0}$, such that $d\left(F\left(x_{0}\right), x_{\circ}\right) \preceq\left(e_{\mathcal{A}}-L\right) r_{0}$, with $\theta \ll r_{\circ} \ll r$. (Otherwise for each $n \in \mathbb{N}$ we have $(1-1 / n)\left(e_{\mathcal{A}}-L\right) r \prec d\left(F\left(x_{\circ}\right), x_{\circ}\right)$ and then $\left(e_{\mathcal{A}}-L\right) r \preceq d\left(F\left(x_{\circ}\right), x_{\circ}\right)$.) We will show that $F: \overline{B\left(x_{\circ}, r_{\circ}\right)} \rightarrow \overline{B\left(x_{\circ}, r_{\circ}\right)}$. 
Let $x \in \overline{B\left(x_{\circ}, r_{\circ}\right)}$, then

$$
\begin{aligned}
d\left(F(x), x_{\circ}\right) & \preceq d\left(F(x), F\left(x_{\circ}\right)\right)+d\left(F\left(x_{\circ}\right), x_{\circ}\right) \\
& \preceq L d\left(x, x_{\circ}\right)+\left(e_{\mathcal{A}}-L\right) r_{\circ} \preceq r_{\circ} .
\end{aligned}
$$

By Theorem 3.3, $F$ has a unique fixed point in $\overline{B\left(x_{\circ}, r_{\circ}\right)} \subseteq B\left(x_{\circ}, r\right)$. Again it is easy to see that $F$ has only one fixed point in $B\left(x_{\circ}, r\right)$.

Before going to the next theorem, we note that in a Banach algebra $\mathcal{A}$ for each $L \in \mathcal{A}$ with $\|L\|<1$ we can assume that $L \ll e_{\mathcal{A}}$. Indeed, if $\|L\|<1$, then $e_{\mathcal{A}}-L$ is invertible and $\sum_{n=0}^{\infty} L^{n}=\frac{e_{\mathcal{A}}}{e_{\mathcal{A}}-L}$. So $\lim _{n \rightarrow \infty}\left\|L^{n}\right\|=0$. Now by Lemma 1.3(c), there exists a natural number $N$, such that $L^{N} \ll e_{\mathcal{A}}$.

Theorem 3.7 Let $C$ be a nonempty, closed, and convex subset of an algebraic cone norm space X. Also let $F: C \rightarrow C$ be a Lipschitzian map with Lipschitz coefficient $L$ such that $\|L\|=1$ and $F(C)$ be a subset of a compact subset of $C$. Then $F$ has a fixed point.

Proof Since $\|L\|=1$ and $d(F(x), F(y)) \preceq L d(x, y)$ for all $x, y \in X$, let $x_{\circ} \in C$ and, for $n=$ $2,3, \ldots$, define

$$
F_{n}(x)=\left(1-\frac{1}{n}\right) F(x)+\frac{1}{n} x_{\circ} .
$$

Since $C$ is convex and $x_{\circ} \in C, F_{n}: C \rightarrow C$ is a contraction. Therefore, by Theorem 3.3 each $F_{n}$ has a unique fixed point $x_{n} \in C$. That is,

$$
x_{n}=F_{n}\left(x_{n}\right)=\left(1-\frac{1}{n}\right) F\left(x_{n}\right)+\frac{1}{n} x_{0} .
$$

In addition, since $F(C)$ lies in a compact subset of $C$, there exists a subsequence $S$ of integers and a $u \in C$ with $\lim _{n \rightarrow \infty} F\left(x_{n}\right)=u$ in $S$. Thus,

$$
x_{n}=\left(1-\frac{1}{n}\right) F\left(x_{n}\right)+\frac{1}{n} x_{0} \rightarrow u \quad \text { as } n \rightarrow \infty
$$

in $S$. So by continuity, $F\left(x_{n}\right) \rightarrow F(u)$ as $n \rightarrow \infty$ in $S$, and therefore $u=F(u)$.

Theorem 3.8 (See Theorem 2.1 in [17]) Let $(X, d)$ be a complete cone metric space, and $\mathcal{P}$ be an algebraic cone. Suppose that the mapping $f$ and $g$ are two self-maps of $X$ satisfying

$$
d(f(x), g(x)) \preceq \alpha d(x, y)+\beta[d(x, f(x))+d(y, g(y))]+\gamma[d(x, g(y))+d(y, f(x))]
$$

for all $x, y \in X$, where $\alpha, \beta, \gamma \in \mathcal{P}$ and

$$
\|\alpha\|+2\|\beta\|+2\|\gamma\|<1, \quad\|\alpha+\beta+\gamma\|<\left\|e_{\mathcal{A}}-(\beta+\gamma)\right\| .
$$

Then $f$ and $g$ have a unique common fixed point in X. Moreover, any fixed point of $f$ is a fixed point of $g$, and vice versa. 
Proof Suppose $x_{\circ}$ is an arbitrary point of $X$ and define $\left\{x_{n}\right\}$ by $x_{2 n+1}=f\left(x_{2 n}\right), x_{2 n+2}=$ $g\left(x_{2 n+1}\right)$ for $n=0,1,2, \ldots$. Then

$$
d\left(x_{2 n+1}, x_{2 n+2}\right) \preceq(\alpha+\beta+\gamma) d\left(x_{2 n}, x_{2 n+1}\right)+(\beta+\gamma) d\left(x_{2 n+1}, x_{2 n+2}\right),
$$

which implies that $d\left(x_{2 n+1}, x_{2 n+2}\right) \preceq \delta d\left(x_{2 n}, x_{2 n+1}\right)$, where

$$
\delta=(\alpha+\beta+\gamma)\left(e_{\mathcal{A}}-(\beta+\gamma)\right)^{-1}
$$

(since $\|\beta+\gamma\|<1, e_{\mathcal{A}}-(\beta+\gamma)$ is invertible). Similarly, it can be shown that

$$
d\left(x_{2 n+3}, x_{2 n+2}\right) \preceq \delta d\left(x_{2 n+2}, x_{2 n+1}\right) .
$$

Therefore, for all $n$ we have

$$
d\left(x_{n+1}, x_{n+2}\right) \preceq \delta^{n+1} d\left(x_{0}, x_{1}\right),
$$

and, hence, for $m>n$,

$$
d\left(x_{m}, x_{n}\right) \preceq\left(\delta^{n}+\delta^{n+1}+\cdots+\delta^{m-1}\right) d\left(x_{1}, x_{\circ}\right) \preceq \delta^{n}\left(e_{\mathcal{A}}-\delta\right)^{-1} d\left(x_{1}, x_{\circ}\right),
$$

so that Lemma 1.3(b) implies $d\left(x_{n}, x_{m}\right) \rightarrow \theta$. Hence $\left\{x_{n}\right\}$ is a Cauchy sequence. Since $X$ is complete, there exists $p \in X$, such that $x_{n} \rightarrow p$. Now by (3.1),

$$
\begin{aligned}
d(p, g(p)) \preceq & d\left(p, x_{2 n+1}\right)+d\left(x_{2 n+1}, g(p)\right) \\
\preceq & d\left(p, x_{2 n+1}\right)+\alpha d\left(x_{2 n}, p\right)+\beta\left[d\left(x_{2 n}, x_{2 n+1}\right)+d(p, g(p))\right] \\
& +\gamma\left[d\left(x_{2 n}, p\right)+d(p, g(p))+d\left(p, x_{2 n+1}\right)\right],
\end{aligned}
$$

and so

$$
\begin{aligned}
d(p, g(p)) \leq & \left(e_{\mathcal{A}}-(\alpha+\beta)\right)^{-1}\left[d\left(p, x_{2 n+1}\right)+\alpha d\left(x_{2 n}, p\right)\right. \\
& \left.+\beta d\left(x_{2 n}, x_{2 n+1}\right)+\gamma\left(d\left(x_{2 n}, p\right)+d\left(p, x_{2 n+1}\right)\right)\right] .
\end{aligned}
$$

Thus $g p=p$, by Lemma 1.3(a). Now, $d(f(p), p) \preceq(\beta+\gamma) d(p, f(p))$, and so

$$
\left(e_{\mathcal{A}}-(\beta+\gamma)\right) d(p, f(p)) \geq \theta
$$

implies $f(p)=p$.

To prove uniqueness, suppose that $q$ is another common fixed point of $f$ and $g$, then $d(p, q) \preceq(\alpha+2 \gamma) d(p, q)$, which gives $d(p, q)=\theta$, and $p=q$.

Theorem 3.9 (See Theorem 2.2 in [7] and Theorem 3.2 in [9]) Let $(X, d)$ be a complete cone metric space and $\mathcal{P}$ be an algebraic cone. Suppose the mapping $T: X \rightarrow X$ satisfies the contraction condition

$$
d(T x, T y) \preceq k(d(T x, x)+d(T y, y))
$$


for all $x, y \in X$, where $k \in \mathcal{P}, \sum_{n=0}^{\infty} k^{n}$ and $\sum_{n=0}^{\infty}\left(k\left(e_{\mathcal{A}}-k\right)^{-1}\right)^{n}$ are absolutely convergent. Then $T$ has a unique fixed point in $X$, and for any $x \in X$, the iterative sequence $\left\{T^{n} x\right\}$ converges to the fixed point.

Proof Choose $x_{0} \in X$ and for each $n \in \mathbb{N}$ set $x_{1}=T x_{0}, \ldots, x_{n+1}=T x_{n}$. Then

$$
\begin{aligned}
d\left(x_{n+1}, x_{n}\right) & =d\left(T x_{n}, T x_{n-1}\right) \\
& \preceq k\left(d\left(T x_{n}, x_{n}\right)+d\left(T x_{n-1}, x_{n-1}\right)\right) .
\end{aligned}
$$

So $\left(e_{\mathcal{A}}-k\right) d\left(x_{n+1}, x_{n}\right) \preceq k d\left(x_{n}, x_{n-1}\right)$. Since $e_{\mathcal{A}}-k$ is invertible, so

$$
d\left(x_{n+1}, x_{n}\right) \preceq k\left(e_{\mathcal{A}}-k\right)^{-1} d\left(x_{n}, x_{n-1}\right) .
$$

Let $h=k\left(e_{\mathcal{A}}-k\right)^{-1}$, then $h$ is invertible. For $n>m$,

$$
\begin{aligned}
d\left(x_{n}, x_{m}\right) & \preceq \sum_{i=m}^{n-1} d\left(x_{i}, x_{i+1}\right) \\
& \preceq \sum_{i=m}^{n-1} h^{i} d\left(x_{0}, x_{1}\right) .
\end{aligned}
$$

Since $\sum_{i=0}^{\infty} h^{i}$ converges to $\left(e_{\mathcal{A}}-h\right)^{-1}$, so $\left\{\sum_{i=0}^{n} h^{i}\right\}_{n=1}^{\infty}$ is a Cauchy sequence. Therefore, $\left\{x_{n}\right\}$ is Cauchy. Since $X$ is complete, there is a $x^{*} \in X$, such that $x_{n} \rightarrow x^{*}$. Thus,

$$
\begin{aligned}
d\left(T x^{*}, x^{*}\right) & \leq d\left(T x_{n}, T x^{*}\right)+d\left(T x_{n}, x^{*}\right) \\
& \preceq k\left(d\left(T x_{n}, x_{n}\right)+d\left(T x^{*}, x^{*}\right)\right)+d\left(x_{n+1}, x^{*}\right) .
\end{aligned}
$$

So $(1-k) d\left(T x^{*}, x^{*}\right) \preceq k d\left(T x_{n}, x_{n}\right)+d\left(x_{n+1}, x^{*}\right)$ and

$$
d\left(T x^{*}, x^{*}\right) \preceq\left(e_{\mathcal{A}}-k\right)^{-1}\left(k d\left(T x_{n}, x_{n}\right)+d\left(x_{n+1}, x^{*}\right)\right) .
$$

Since $d$ is continuous, so $d\left(T x^{*}, x^{*}\right) \preceq \theta$ and hence $T x^{*}=x^{*}$.

Now, let $y^{*}$ be another fixed point of $T$, then

$$
\begin{aligned}
d\left(x^{*}, y^{*}\right) & =d\left(T x^{*}, T y^{*}\right) \\
& \preceq k\left(d\left(T x^{*}, x^{*}\right)+d\left(T y^{*}, y^{*}\right)\right)=\theta .
\end{aligned}
$$

Hence $x^{*}=y^{*}$.

Remark 3.10 In Theorem 3.9 if $\|k\| \cdot \frac{1}{\left\|e_{\mathcal{A}}-k\right\|}<1$, then the series $\sum_{n=0}^{\infty} k^{n}$ and $\sum_{n=0}^{\infty}\left(k\left(e_{\mathcal{A}}-\right.\right.$ $\left.k)^{-1}\right)^{n}$ show absolute convergence.

Theorem 3.11 Let $(X, d)$ be a complete algebraic cone metric space, $q \in \mathcal{P}, \sum_{n=1}^{\infty} q^{n}$ be absolutely convergent and $T: X \rightarrow X$ be a function. If for each $x \in X$ there exists a positive integer $n=n(x)$, such that

$$
d\left(T^{n} x, T^{n} y\right) \preceq q \cdot v
$$


for some

$$
v \in\left\{d(x, y), d(x, T y), d\left(x, T^{2} y\right), \ldots, d\left(x, T^{n} y\right), d\left(x, T^{n} x\right)\right\}
$$

and for all $y \in X$, then $T$ has a unique fixed point $u \in X$. Moreover, for every $x \in X, u=$ $\lim _{m \rightarrow \infty} T^{m} x$.

Proof The proof is similar to Theorem 3.1 in [18].

\section{Examples}

In this section, $\mathbb{C}^{X}$ and $C(X)$, will denote the set of all complex-valued and the set of all continuous complex-valued functions on a topological space $X$, respectively. There are several natural ways of introducing a topology on $\mathbb{C}^{X}$ and $C(X)$ and they all follow the general approach indicated below.

Let $S$ be a family of subsets of $X$. For each $P \in S$ and each $U$ of open subset of $\mathbb{C}$, let $M(P, U)$ consisting the set of all $f \in \mathbb{C}^{X}$ for which $f(P) \subseteq U$. Put $O(P, U)=M(P, U) \cap C(X)$. Sets of this form are a subbase of some topology on $\mathbb{C}^{X}$, or similarity on $C(X)$, called the topology of convergence with respect to elements of $S$. Under this construction, the space $C(X)$ is a subspace of the space $\mathbb{C}^{X}$. The most important topologies obtained in this way are the following: the topology of pointwise convergence, corresponding to the case when $S$ consists of all finite subsets of $X$, and the compact-open topology, arising when $S$ consists of all compact subsets of the space $X$. The topologies of uniform convergence on $\mathbb{C}^{X}$ and $C(X)$ are described in slightly different ways. Their bases consist of the sets $U^{\prime}(f, \epsilon)=\{g \in$ $\left.\mathbb{C}^{X}: \sup _{x \in X}|f(x)-g(x)|<\epsilon\right\}$ and $U(f, \epsilon)=C(X) \cap U^{\prime}(f, \epsilon)$, respectively. If $X$ is a completely regular compact space, then the topology of uniform convergence and the compact-open topology are equal on $C(X)$.

(a) Let $\mathbb{C}^{X}$ be equipped with the pointwise topology. So in $\mathbb{C}^{X}$, a sequence $\left\{f_{n}\right\}_{n \in \mathbb{N}} \subseteq$ $\mathbb{C}^{X}$ converges to $g \in \mathbb{C}^{X}$ if and only if $\left\{f_{n}(x)\right\}_{n \in \mathbb{N}}$ converges to $g(x)$ for each $x$ in $X$. It is obvious that $\mathbb{C}^{X}$ under the pointwise topology is a topological algebra (see the definitions in Section 2). Define

$$
\mathcal{P}=\left\{f \in \mathbb{C}^{X}: f(x) \geq 0 \text { for each } x \in X\right\} .
$$

It is obvious that $\mathcal{P}$ is an algebraic cone. Now define

$$
\langle\cdot, \cdot\rangle: \mathbb{C}^{X} \times \mathbb{C}^{X} \rightarrow \mathbb{C}^{X}
$$

by $\langle f, g\rangle=f \bar{g}$ for each $f, g \in \mathbb{C}^{X}$. It is obvious that $\langle\cdot, \cdot\rangle$ has the following properties:

(i) $\langle\alpha f+h, g\rangle=\alpha\langle f, g\rangle+\langle h, g\rangle$ for each $f, g, h \in \mathbb{C}^{X}$ and each $\alpha \in \mathbb{C}$.

(ii) $\langle f, \alpha g+h\rangle=\bar{\alpha}\langle f, g\rangle+\langle h, g\rangle$ for each $f, g, h \in \mathbb{C}^{X}$ and each $\alpha \in \mathbb{C}$.

(iii) $\langle f, g\rangle=\overline{\langle g, f\rangle}$ for each $f, g \in \mathbb{C}^{X}$.

(iv) $\langle f, f\rangle=0$ if and only if $f=0$.

$\langle\cdot, \cdot\rangle$ is a vector-valued inner product on $\mathbb{C}^{X}$. Now define $\|f\|_{\mathcal{P}}=\langle f, f\rangle^{\frac{1}{2}}=|f|$. It is obvious that $\|\cdot\|_{\mathcal{P}}$ is a cone algebraic norm on $\mathcal{P}$. It is obvious that int $\mathcal{P}=\left\{f \in \mathbb{C}^{X}: f(x)>\right.$ 0 for each $x \in X\}$. $\left(\mathbb{C}^{X},\|\cdot\|_{\mathcal{P}}\right)$ is a Banach cone algebra. For this purpose, we prove that $\left(\mathbb{C}^{X},\|\cdot\|_{\mathcal{P}}\right)$ is complete. Let $\left\{f_{n}\right\}_{n=1}^{\infty}$ be a Cauchy sequence, then for each $x \in X,\left\{f_{n}(x)\right\}$ is a 
Cauchy sequence in $\mathbb{C}$. Therefore, there exists $g \in \mathbb{C}^{X}$, such that $\left\{f_{n}\right\}$ is pointwise convergent to $g$. This shows that $\left(\mathbb{C}^{X},\|\cdot\|_{\mathcal{P}}\right)$ is complete.

(b) Let $(X, \tau)$ be a completely regular non-pseudocompact topological space. Let $C(X)$ be equipped with pointwise convergence topology. It is obvious that $C(X)$ is a topological subalgebra of $\mathbb{C}^{X}$. But $C(X)$ is not a cone complete subalgebra of $\mathbb{C}^{X}$, because $C(X)$ is not closed.

(c) Let $(X, \tau)$ be a completely regular non-pseudocompact topological space. Let $C(X)$ be equipped with uniform convergence topology. A sequence $\left\{f_{n}\right\}_{n \in \mathbb{N}} \subseteq C(X)$ converges to $g \in C(X)$ if and only if $\left\{f_{n}\right\}_{n \in \mathbb{N}}$ uniformly converges to $g$ on $X$. $C(X)$ under uniform convergence on $X$ is not a topological algebra, because multiplication is not continuous.

(d) Let $(X, \tau)$ be a completely regular non-pseudocompact topological space. Let $C(X)$ be equipped with compact-open topology. A sequence $\left\{f_{n}\right\}_{n \in \mathbb{N}} \subseteq C(X)$ converges to $g \in C(X)$ if and only if $\left\{f_{n}\right\}_{n \in \mathbb{N}}$ uniformly converges to $g$ on every compact subset of $X$. It is obvious that $\mathbb{C}^{X}$ under the compact-open topology is a topological algebra. Define

$$
\mathcal{P}=\{f \in C(X): f(x) \geq 0 \text { for each } x \in X\} .
$$

It is obvious that $\mathcal{P}$ is a cone. Now define

$$
\langle\cdot, \cdot\rangle: C(X) \times C(X) \rightarrow C(X)
$$

by $\langle f, g\rangle=f \bar{g}$ for each $f, g \in C(X)$. It is obvious that $\langle\cdot, \cdot\rangle$ has the following properties:

(i) $\langle\alpha f+h, g\rangle=\alpha\langle f, g\rangle+\langle h, g\rangle$ for each $f, g, h \in C(X)$ and each $\alpha \in \mathbb{C}$.

(ii) $\langle f, \alpha g+h\rangle=\bar{\alpha}\langle f, g\rangle+\langle h, g\rangle$ for each $f, g, h \in C(X)$ and each $\alpha \in \mathbb{C}$.

(iii) $\langle f, g\rangle=\overline{\langle g, f\rangle}$ for each $f, g \in C(X)$.

(iv) $\langle f, f\rangle=0$ if and only if $f=0$.

$\langle\cdot, \cdot\rangle$ is a vector-valued inner product on $C(X)$. Now define $\|f\|_{c}=\langle f, f\rangle^{\frac{1}{2}}=|f|$. It is obvious that $\|\cdot\|_{c}$ is a cone algebraic norm on $\mathcal{P}$. It is obvious that int $\mathcal{P}=\{f \in C(X): f(x)>$ 0 for each $x \in X\} .\left(C(X),\|\cdot\|_{c}\right)$ is a Banach cone algebra. For this we prove that $\left(C(X),\|\cdot\|_{c}\right)$ is complete. Let $\left\{f_{n}\right\}_{n=1}^{\infty}$ be a Cauchy sequence, then, for each compact subset of $X,\left\{f_{n}\right\}_{n \in \mathbb{N}}$ is uniformly Cauchy, so for each $x \in X\left\{f_{n}\right\}$ is uniformly convergent. Therefore, there exists $g \in C(X)$ such that $\left\{f_{n}\right\}$ is compact-open convergent to $g$. This shows that $\left(C(X),\|\cdot\|_{c}\right)$ is complete.

(e) Let $\mathcal{A}=C_{\mathbb{R}}^{1}[0,1]$ with the norm $\|f\|=\|f\|_{\infty}+\left\|f^{\prime}\right\|_{\infty}$ and $\mathcal{P}=\{f \in \mathcal{A}: f(x)>$ 0 on $[0,1]\}$, that is, a non-normal solid cone ([2], p.221). We note that:

(1) $\left(C_{\mathbb{R}}^{1}[0,1],\|\cdot\|\right)$ with pointwise operations is a (cone) Banach algebra.

(2) Consider $f_{n}(x)=\frac{x^{n}}{n}$ for $n \in \mathbb{N}$ and $x \in[0,1]$ and let $X=\left\{f_{n}: n \in \mathbb{N}\right\} \cup\{0\}$, where 0 is the constant function zero. Further, define the cone metric $d: X \times X \rightarrow \mathcal{A}$ by $d(f, g)=|f-g|$. Then $\lim _{n \rightarrow \infty} f_{n}=0$ in the cone metric space $(X, d)$, however, $\left\|d\left(f_{n}, 0\right)\right\|=1+\frac{1}{n} \rightarrow 1$. Therefore $(X, d)$ is a Banach cone metric space with an algebraic non-normal cone.

(3) Suppose $X$ is as (2) and define $d^{\prime}: X \times X \rightarrow \mathcal{A}$ by $d^{\prime}(f, g)=f+g, x \neq y$ and $d^{\prime}(f, f)=0$. Then $d^{\prime}$ is a cone metric space too and for $g_{n}(x)=\frac{1-\sin n x}{n+2}$ and $h_{n}(x)=\frac{1+\sin n x}{n+2}$, since $0 \preceq g_{n} \preceq g_{n}+h_{n} \rightarrow 0$, we have $d\left(g_{n}, 0\right) \rightarrow 0$ in the cone metric space $\left(X, d^{\prime}\right)$, but $\left\|d\left(g_{n}, 0\right)\right\|=g_{n} \nrightarrow 0$. Therefore $\left(X, d^{\prime}\right)$ is a Banach cone metric space with a non-continuous metric. 
(f) This example was first treated by Kaplansky for another purpose. Let $\left\{z_{n}\right\}_{n \in \mathbb{N}}$ be the natural orthonormal basis of $l^{2}(\mathbb{N})$. The weighted shift operators defined by

$$
S\left(z_{m}\right)=s_{m} z_{m+1} \quad(m \in \mathbb{N})
$$

for some bounded sequence $\left\{s_{m}\right\}_{m \in \mathbb{N}}$ of weights (see [19], Example 2.2.16). The norm of such an operator is easily seen to be

$$
\|S\|=\sup \left\{\left|s_{m}\right|: m \in \mathbb{N}\right\} .
$$

For each $m, n \in \mathbb{N}$, define $t_{m}=\left(\frac{2 m-1}{2 m}\right)^{3}$ and $T\left(z_{m}\right)=t_{m} z_{m+1}$ for each $m \in \mathbb{N}$. The norm of $T^{n}$ is given by

$$
\left\|T^{n}\right\|=\sup \left\{\prod_{m=k}^{k+n-1} t_{m}: k \in \mathbb{N}\right\}
$$

So $\left\|T^{n}\right\|=\left(\frac{1 \cdot 3 \cdots 2 n-1}{2 \cdot 4 \cdots 2 n}\right)^{3}$. This implies that $\sum_{n=1}^{\infty}\left\|T^{n}\right\|=\sum_{n=1}^{\infty}\left(\frac{1 \cdot 3 \cdots 2 n-1}{2 \cdot 4 \cdots 2 n}\right)^{3}$ is convergent and $\limsup _{n \rightarrow \infty}\left\|T^{n}\right\|=1$.

\section{Competing interests}

The authors declare that they have no competing interests.

\section{Authors' contributions}

All authors contributed equally to the writing of this paper. All authors read and approved the final manuscript.

\section{Author details}

${ }^{1}$ Department of Mathematics, Shahed University, Tehran, Iran. ${ }^{2}$ Department of Mathematics, Qom University, Qom, Iran.

\section{Acknowledgements}

The authors are very grateful to the anonymous referee for his or her comments and suggestions.

Received: 5 March 2014 Accepted: 14 June 2014 Published: 23 Jul 2014

\section{References}

1. Huang, LG, Zhang, X: Cone metric spaces and fixed point theorems of contractive mapping. J. Math. Anal. Appl. 332(2), 1468-1476 (2007)

2. Deimling, K: Nonlinear Functional Analysis. Springer, Berlin (1985)

3. Janković, S, Kadelburg, Z, Radenović, S: On cone metric spaces: a survey. Nonlinear Anal. 74, 2591-2601 (2011)

4. Krein, M: Propriétés fondamentales des ensembles coniques normaux dans l'espace de Banach. Dokl. Akad. Nauk SSSR 28, 13-17 (1940)

5. Wong, Y-C, Ng, K-F: Partially Ordered Topological Vector Spaces. Clarendon, Oxford (1973)

6. Popović, B, Radenović, S, Shukla, S: Fixed point results to tvs-cone b-metric spaces. Gulf J. Math. 1, 51-64 (2013)

7. Liu, H, Xu, S: Cone metric spaces with Banach algebras and fixed point theorems of generalized Lipschitz mappings. Fixed Point Theory Appl. 2013, 320 (2013)

8. Liu, H, Xu, S: Fixed point theorem of quasi-contractions on cone metric spaces with Banach algebras. Abstr. Appl. Anal. 2013, Article ID 187348 (2013)

9. Xu, S, Radenović, S: Fixed point theorems of generalized Lipschitz mappings on cone metric spaces over Banach algebras without assumption of normality. Fixed Point Theory Appl. 2014, 102 (2014)

10. Abdeljawad, T: Completion of cone metric spaces. Hacet. J. Math. Stat. 39(1), 67-74 (2010)

11. Abdeljawad, T, Turkoglu, D, Abuloha, M: Some theorems and examples of cone metric spaces. J. Comput. Anal. Appl. 12(4), 739-753 (2010)

12. Karapinar, E: Fixed point theorems in cone Banach spaces. Fixed Point Theory Appl. 2009, Article ID 609281 (2009). doi:10.1155/2009/609281

13. Asadi, M, Veezpour, SM, Rhoodes, BE, Soleimani, H: Metrizability of cone metric spaces via renorming the Banach spaces. J. Nonlinear Anal. Appl. 2012, Article ID jnaa-00160 (2012)

14. Rudin, W: Functional Analysis. Springer, Berlin (1970)

15. Kadelburg, Z, Radenović, S: A note on various types of cones and fixed point results in cone metric spaces. Asian J. Math. Appl. 2013, Article ID ama0104 (2013)

16. Agarwal, RP, Meehan, M, O'Regan, D: Fixed Point Theory and Application. Cambridge University Press, Cambridge (2004)

17. Abbas, M, Rhoades, BE: Fixed and periodic point results in cone metric spaces. Appl. Math. Lett. 22, $511-515$ (2009) 
18. Gajić, L, llić, D, Rakočević, V: On Ćirić maps with a generalized contractive iterate at a point and Fisher's quasicontractions in cone metric spaces. Appl. Math. Comput. 216, 2240-2247 (2010)

19. Palmer, TW: Banach Algebras and the General Theory of *-Algebras. Volume 1: Algebras and Banach Algebras. Cambridge University Press, Cambridge (1994)

10.1186/1687-1812-2014-160

Cite this article as: Tootkaboni and Bagheri Salec: Algebraic cone metric spaces and fixed point theorems of contractive mappings. Fixed Point Theory and Applications 2014, 2014:160

Submit your manuscript to a SpringerOpen ${ }^{\circ}$ journal and benefit from:

- Convenient online submission

Rigorous peer review

- Immediate publication on acceptance

Open access: articles freely available online

- High visibility within the field

- Retaining the copyright to your article 